\title{
国国pubvet
}

https://doi.org/10.31533/pubvet.v14n2a504.1-6

\section{Tratamento cirúrgico do glaucoma por ciclofotocoagulação a laser em cães}

\author{
Lilian Santos Lage ${ }^{\bullet}$, Luiz Fernando Lucas Ferreira ${ }^{2 *}{ }^{\bullet}$, Amanda Ladeia Fernandes ${ }^{3}$, Bruna $^{\circ}$ \\ Ferraz Calhau ${ }^{30}$
}

${ }^{I}$ Médica veterinária, graduada pela Pontifícia Universidade Católica de Minas Gerais (PUC Minas-Betim).

${ }^{2}$ Médico Veterinário e Professor da Pontifícia Universidade Católica de Minas Gerais, Departamento de Medicina Veterinária, Betim - MG Brasil. ${ }^{3}$ Graduanda em Medicina Veterinária, Pontifícia Universidade Católica de Minas Gerais, (PUC Minas-Betim)

*Autor para correspondência, E-mail: Ifvet@hotmail.com

Resumo. O glaucoma é uma das oftalmopatias mais importantes da atualidade, sendo uma das doenças oculares mais estudadas no mundo. Porém, apesar de tantos estudos, até hoje não há um consenso entre os médicos veterinários sobre qual o melhor tratamento para esta afecção. Com os avanços da tecnologia, como o surgimento do laser oftalmológico, novas técnicas para o tratamento cirúrgico do glaucoma foram criadas, sendo cada vez mais eficazes menos invasivas e com menos complicações pós-operatórias. Um exemplo de uma dessas técnicas relativamente novas é a ciclofotocoagulação a laser, que tem demonstrado ser uma das mais eficazes para o controle da pressão intraocular (PIO). Porém, essa técnica ainda foi pouco estudada. No presente trabalho foi realizado um experimento em oito cães diagnosticados com glaucoma, totalizando onze olhos, com o objetivo de avaliar a eficácia da ciclofotocoagulação a laser no tratamento do glaucoma. Houve diminuição da PIO em $100 \%$ dos cães operados e a técnica mostrou-se eficaz, mantendo a PIO entre os valores de normalidade em $55 \%$ dos casos.

Palavras-chave: cão, ciclofotocoagulação, glaucoma, tratamento

\section{Surgical treatment of glaucoma by laser cyclophotocoagulation in dogs}

\begin{abstract}
Glaucoma is one of the important eye diseases today, one of the most studied eye diseases in the world. However, despite many studies, to date there is no consensus among veterinarians about the best treatment for this medical condition. With advances in technology, such as the emergence of ophthalmic laser, new techniques for the surgical treatment of glaucoma were developed, which are increasingly more effective, less invasive, and causing less postoperative complications. An example is the relatively new cyclophotocoagulation laser, which has proven to be one of the most effective techniques for IOP control. However, there have been few studies on this technique. An experiment using eight glaucomatous dogs - total of eleven eyes - was carried out to evaluate the effectiveness of laser cyclophotocoagulation as a treatment for glaucoma. This experiment shows a decreased in IOP in 100\% of the operated dogs, and the technique has been proven effective, maintaining normal IOP levels in 55\% of the cases.
\end{abstract}

Keywords: cyclophotocoagulation, dog, glaucoma, treatment

\section{Tratamiento quirúrgico del glaucoma de ciclofotocoagulación con láser en perros}

Resumen. El glaucoma es una de las oftalmopatías más importantes en la actualidad, siendo una de las enfermedades oculares más estudiadas del mundo. Sin embargo, a pesar 
de tantos estudios, hasta la fecha no hay consenso entre los veterinarios sobre el mejor tratamiento para esta afección. Con los avances tecnológicos, como la aparición del láser oftálmico, se han creado nuevas técnicas para el tratamiento quirúrgico del glaucoma que son cada vez más efectivas, menos invasivas y con menos complicaciones postoperatorias. Un ejemplo de una de estas técnicas relativamente nuevas es la ciclofotocoagulación con láser, que ha demostrado ser una de las más efectivas para controlar la preción intraocular (PIO). Sin embargo, esta técnica no ha sido muy estudiada. En el presente trabajo, se realizó un experimento en ocho perros diagnosticados con glaucoma, con un total de once ojos, con el objetivo de evaluar la efectividad de la ciclofotocoagulación con láser en el tratamiento del glaucoma. La PIO disminuyó en el 100\% de los perros operados y la técnica fue efectiva, manteniendo la PIO entre los valores normales en el 55\% de los casos.

Palabras claves: ciclofotocoagulación, perro, glaucoma, tratamiento

\section{Introdução}

A oftalmologia veterinária é uma especialidade que se encontra em constante expansão, em razão do aumento do diagnóstico das oftalmopatias em cães, que, devido aos avanços da medicina veterinária, tiveram sua expectativa de vida aumentada. Com isso, é de grande importância para os médicos veterinários o conhecimento das doenças oculares que são mais frequentemente encontradas na clínica de pequenos animais. Entre essas se destaca o glaucoma, que é uma síndrome que acomete animais de diferentes espécies e em diferentes momentos de sua vida, tendo causas variáveis. É uma emergência oftalmológica sendo considerada uma das maiores causas de perda de visão em cães, tendo a ocorrência de aproximadamente $0,8 \%$ na população canina. A definição de glaucoma tem evoluído nas últimas décadas. Assim como as formas de tratá-lo, que ainda é um assunto que gera discussão entre os médicos veterinários.

Como os primeiros sinais do glaucoma passam despercebidos pelos proprietários, ao ser diagnosticado, geralmente já se encontra avançado, dificultando o sucesso do tratamento, pois na maioria das vezes os indicativos da doença só são notados quando a pressão intraocular (PIO) já se encontra muito elevada.

Atualmente existem várias formas de tratamento do glaucoma, medicamentoso e/ou cirúrgico. Porém, não há um consenso entre os profissionais sobre a melhor forma de tratá-lo. Primariamente a terapia de escolha é a medicamentosa, através de fármacos mióticos, inibidores de anidrase carbônica, agentes colinérgicos, análogos da prostaglandina ou hiperosmóticos. Mas, sabe-se que, em alguns casos, o glaucoma torna-se refratário à terapia medicamentosa ao longo do tratamento, e a intervenção cirúrgica faz-se obrigatória para o alívio da dor e do desconforto ocular.

Neste experimento a técnica cirúrgica para o tratamento do glaucoma que será discutida é a ciclofotocoagulação a laser, utilizando laser diodo; por ser uma cirurgia pouco estudada e realizada aqui no Brasil, é mais fácil de ser executada, menos invasiva, menos traumática, causa menos danos teciduais e menos processos inflamatórios.

Este experimento teve como objetivo avaliar qualitativamente e quantitativamente a eficácia da ciclofotocoagulação a laser no controle da PIO para o tratamento do glaucoma canino.

\section{Metodologia}

Foram utilizados oito cães, de raças variadas, sendo cinco machos e três fêmeas, portadores de glaucoma crônico uni ou bilateral. O grupo foi composto por cães atendidos pelo serviço de oftalmologia da Clínica Veterinária Professor Israel, situada na cidade de Belo Horizonte/MG.

Os cães do presente estudo foram previamente submetidos ao exame oftalmológico de rotina e quando houve a constatação do aumento da PIO, acima de $30 \mathrm{~mm} / \mathrm{Hg}$, ou seja, após o diagnóstico do glaucoma, foi oferecido aos proprietários o tratamento cirúrgico.

A tonometria realizada foi a de aplanação, utilizando-se o tonômetro, próprio para cães, da marca Tonopen ${ }^{\circledR}$, modelo Avia. 
Por ser este estudo um experimento, uma ficha de cadastro e consentimento do procedimento foi elaborada. Os animais foram submetidos aos exames de rotina pré-operatórios: hemograma completo, bioquímica sanguínea completa e eletrocardiograma.

Para o procedimento cirúrgico, os cães receberam como medicação pré-anestésica (MPA) xilazina $2 \%$ (Dopaser - cloridrato de xilazina $2 \%$ - Hertape Calier, Brasil), na dose de $0,5 \mathrm{mg} / \mathrm{kg} / \mathrm{IM}$ associada à morfina (Dimorf - sulfato de morfina - Cristália, Brasil), na dose de $0,2 \mathrm{mg} / \mathrm{kg} / \mathrm{IM}$, aplicadas na mesma seringa. Como anestésico local, foi utilizado colírio de tetracaína, instilando uma gota a cada trinta segundos num total de três gotas, previamente ao início da cirurgia e da antissepsia local com solução tópica de povidine e solução fisiológica de $\mathrm{NaCl}$ 0,9\% estéril (Solução de Cloreto de Sódio 0,9\% - Laboratório Sanobiol Ltda., São Paulo, São Paulo, Brasil).

Após a MPA foi providenciado acesso da veia cefálica direita com cateter 22G (Cateter Angiocath BD - 22G - São Paulo - São Paulo, Brasil) e manutenção de fluidoterapia com solução de cloreto de sódio a $0,9 \%$, em infusão calculada pela dose de $10 \mathrm{ml} / \mathrm{kg} / \mathrm{h}$. A indução anestésica foi feita logo após a realização do acesso venoso com propofol (Propovan - propofol 1\% - Cristália, Brasil) na dose de 2,0 a $4,0 \mathrm{mg} / \mathrm{kg} / \mathrm{IV}$, observando-se perda de reflexos e permitindo a intubação orotraqueal.

A manutenção anestésica foi feita com isoflurano (Forane - isolflurano - Abbott, Brasil), diluído em oxigênio $100 \%$ pelo uso de vaporizador universal, em circuito de reinalação parcial de gases, utilizandose para controle da profundidade os planos de Guedel.

O procedimento cirúrgico foi realizado com aparelho de laser cirúrgico, THERAVET, regulado para realizar pulso único de radiação de laser na potência de $9000 \mathrm{~mW}$ (Figura 1). Os disparos de laser foram realizados em contato com a esclera a aproximadamente $3 \mathrm{~mm}$ do limbo, evitando-se as posições de $3 \mathrm{~h}$ e 9h para não correr o risco de acertar as artérias ciliares (Figura 2). Foram dados doze tiros em cada olho operado (Figura 3).

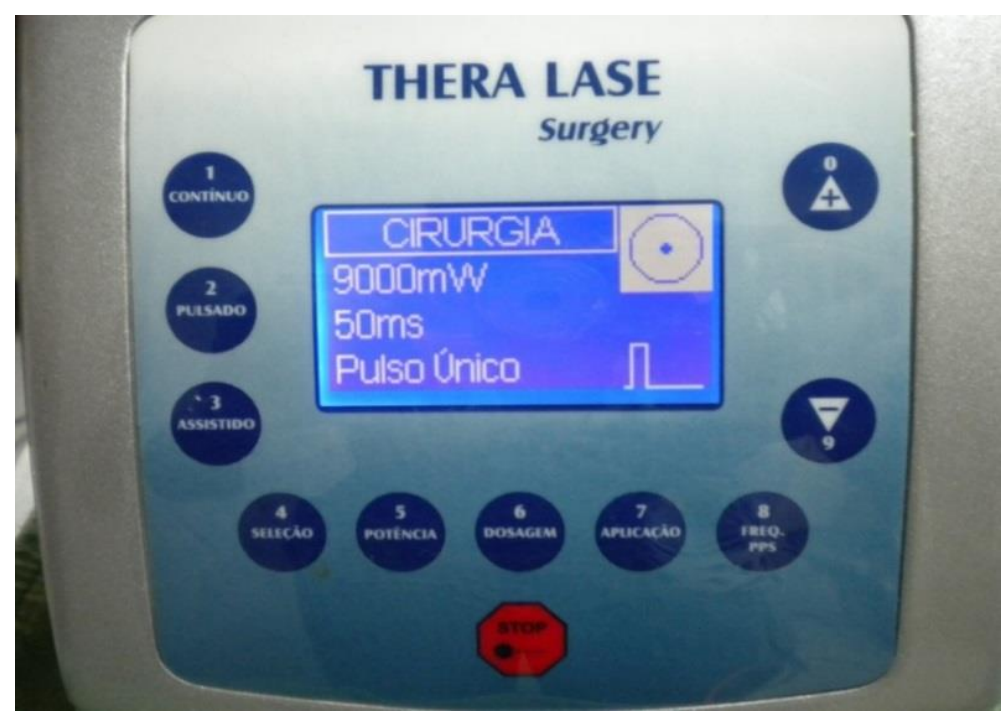

Figura 1. Laser Thera Lase Surgery utilizado durante o experimento para realização da ciclofotocoagulação a laser em cães. Fonte: Arquivo pessoal

O pós-operatório constituiu-se de colírio antiglaucomatoso cloridrato de dorzolamida, duas vezes ao dia, e antibiótico clorofenicol, quatro vezes ao dia durante sete dias.

Houve recomendação da utilização de colar elisabetano para evitar possível lesão ocular causada pelo esfregar dos olhos. Os pacientes fizeram retornos clínicos a cada sete dias para avaliação da pressão intraocular, num total de três retornos.

Os cães que obtiveram valor da PIO normalizada no primeiro retorno foram dispensados do uso dos colírios e assim permaneceram desde que os valores da PIO estiveram normais nos demais retornos.

Para análise estatística dos valores da pressão intraocular antes e após o procedimento cirúrgico utilizou-se o Teste de Wilcoxon para diferença entre pares ordenados. 


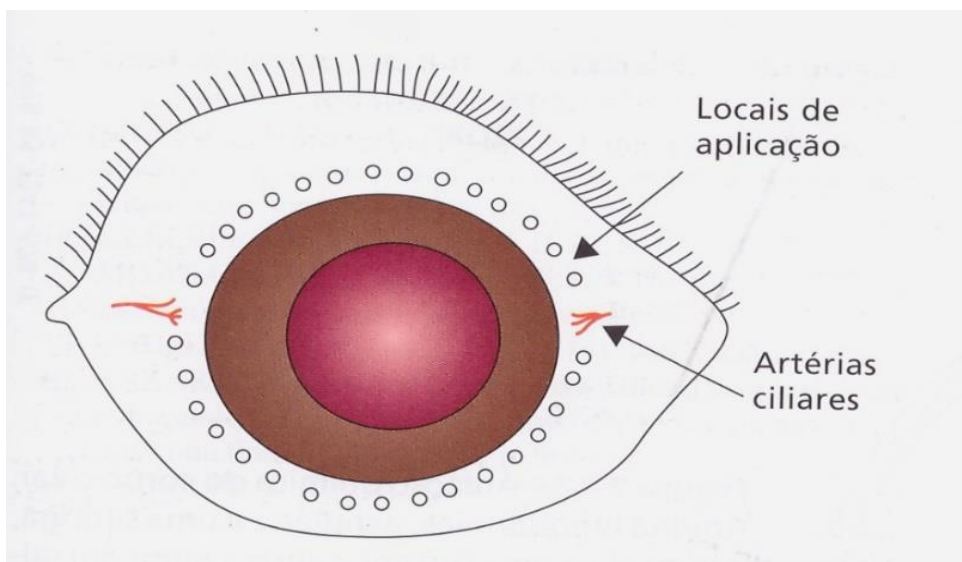

Figura 2. Locais de aplicação do laser diodo para ciclofotocoagulação a laser, e locais a serem evitados por causa das artérias ciliares. Fonte: Laus, 2009.

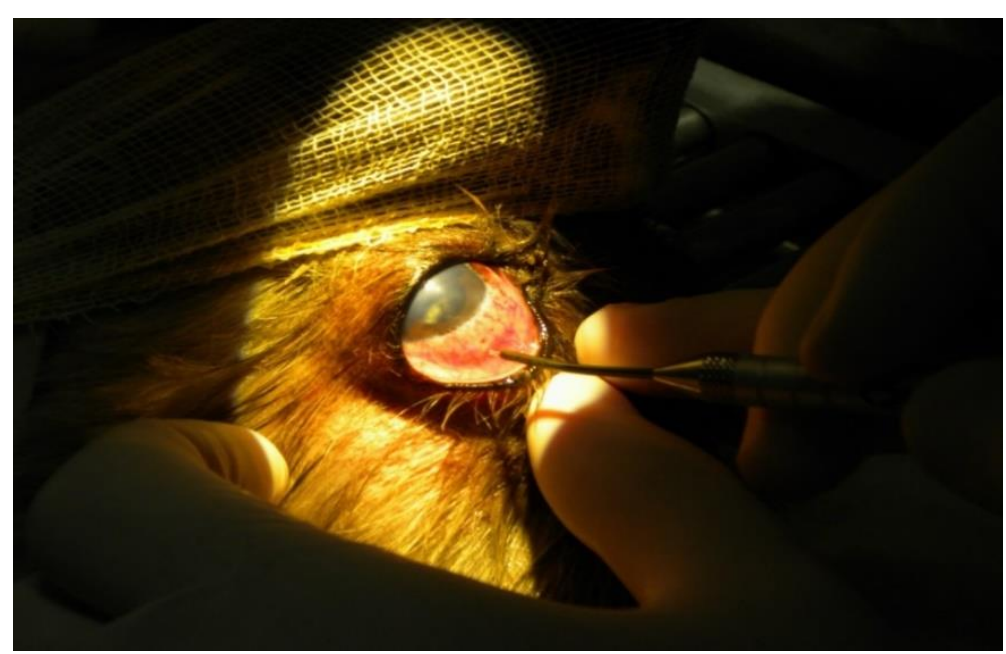

Figura 3. Disparo de laser diodo em olho de cão com glaucoma crônico.

\section{Resultado e discussão}

Durante o experimento oito cães passaram pelo processo cirúrgico de ciclofotocoagulação a laser, totalizando 11 olhos operados, para a verificação da eficácia deste procedimento, como demonstrado no Tabela 1. Foram considerados bem sucedidos os olhos que tiveram redução da pressão intraocular abaixo de $30 \mathrm{mmHg}$ e acima de $15 \mathrm{mmHg}$. Dos 11 olhos operados os 11 tiveram diminuição da PIO após a cirurgia na ordem de $57,15 \%$, como demonstrado no Quadro 1. Os animais que não tiveram a PIO diminuída abaixo de $30 \mathrm{mmHg}$, no caso 3 olhos, o que corresponde a 27,27\%, estão sendo tratados com colírios antiglaucomatosos. De acordo com o Teste de Wilcoxon houve diferença significativa (P $<0,05)$ nas PIO antes e depois da cirurgia, tanto no olho esquerdo quanto no olho direito.

Tabela 1. Informações coletadas sobre o sexo, raça e olho acometido dos cães utilizados no experimento, nas quais OE significa olho esquerdo e OD significa olho direito.

\begin{tabular}{lccc}
\hline Cão & Sexo & Raça & Olho acometido \\
\hline C1 & Macho & Lhasa Apso & OE \\
C2 & Macho & Shar Pei & OD e OE \\
C3 & Fêmea & Poodle & OE \\
C4 & Fêmea & Poodle & OE \\
C5 & Macho & Labrador Retriever & OD \\
C6 & Macho & Dachshund & OD \\
C7 & Macho & Shih Tzu & OE e OD \\
C8 & Fêmea & Poodle & OD e OE \\
\hline
\end{tabular}


Nos estudos realizados por Hardman \& Stanley (2001) e Sapienza \& Van Der Woerdt (2005) os cães utilizados foram diagnosticados com glaucoma primário. Neste experimento, devido à falta de equipamento necessário para diferenciação de glaucoma primário do glaucoma secundário, os cães selecionados foram somente diagnosticados com glaucoma levando em consideração a pressão intraocular acima de $30 \mathrm{mmHg}$, sem ser feita a diferenciação dos tipos de glaucoma. Devido a esse fato, pode haver diferenças estatísticas nos resultados obtidos neste experimento, quando comparando aos de Hardman \& Stanley (2001) e Sapienza \& Van Der Woerdt (2005).

Quaro 1. Aferição da PIO pré e pós-cirúrgica dos animais utilizados durante o experimento, na qual OE significa olho esquerdo e OD significa olho direito.

\begin{tabular}{|c|c|c|c|c|c|c|c|c|c|}
\hline \multirow{2}{*}{ Cão } & \multicolumn{2}{|c|}{ Pio pré-cirúrgico } & \multirow{2}{*}{ Retorno } & \multicolumn{2}{|c|}{ Pio pós-cirúrgico } & \multicolumn{2}{|c|}{ Média pio pós-cirúrgico } & \multicolumn{2}{|c|}{ Percentual } \\
\hline & OD & $\mathrm{OE}$ & & OD & $\mathrm{OE}$ & OD & $\mathrm{OE}$ & OD & $\mathrm{OE}$ \\
\hline \multirow{3}{*}{$\mathrm{C} 1$} & \multirow{3}{*}{$18 *$} & \multirow{3}{*}{73} & 1 & 18 & 33 & \multirow{3}{*}{18,00} & \multirow{3}{*}{33,33} & \multirow{3}{*}{$0,00 \%$} & \multirow{3}{*}{$-54,34 \%$} \\
\hline & & & 2 & 18 & 34 & & & & \\
\hline & & & 3 & 18 & 33 & & & & \\
\hline \multirow{3}{*}{$\mathrm{C} 2$} & \multirow{3}{*}{57} & \multirow{3}{*}{69} & 1 & 29 & 28 & \multirow{3}{*}{29,00} & \multirow{3}{*}{28,33} & \multirow{3}{*}{$-49,12 \%$} & \multirow{3}{*}{$-58,94 \%$} \\
\hline & & & 2 & 29 & 29 & & & & \\
\hline & & & 3 & 29 & 28 & & & & \\
\hline \multirow{3}{*}{$\mathrm{C} 3$} & \multirow{3}{*}{$21^{*}$} & \multirow{3}{*}{62} & 1 & 22 & 32 & \multirow{3}{*}{22,33} & \multirow{3}{*}{27,67} & \multirow{3}{*}{$6,35 \%$} & \multirow{3}{*}{$-55,38 \%$} \\
\hline & & & 2 & 22 & 40 & & & & \\
\hline & & & 3 & 23 & 11 & & & & \\
\hline \multirow{3}{*}{$\mathrm{C} 4$} & \multirow{3}{*}{$*$} & \multirow{3}{*}{57} & 1 & & 15 & \multirow{3}{*}{0,00} & & & \\
\hline & & & 2 & & 16 & & 15,33 & & $-73,10 \%$ \\
\hline & & & 3 & & 15 & & & & \\
\hline & & & 1 & 45 & 15 & & & & \\
\hline $\mathrm{C} 5$ & 74 & $15^{*}$ & 2 & 44 & 16 & 44,67 & 15,67 & $-39,64 \%$ & $4,44 \%$ \\
\hline & & & 3 & 45 & 16 & & & & \\
\hline & & & 1 & 25 & 19 & & & & \\
\hline C6 & 56 & $19 *$ & 2 & 26 & 20 & 25,33 & 19,33 & $-54,76 \%$ & $1,75 \%$ \\
\hline & & & 3 & 25 & 19 & & & & \\
\hline & & & 1 & 18 & 12 & & & & \\
\hline $\mathrm{C} 7$ & 50 & 50 & 2 & 19 & 13 & 18,33 & 12,67 & $-63,33 \%$ & $-74,67 \%$ \\
\hline & & & 3 & 18 & 13 & & & & \\
\hline & & & 1 & 32 & 20 & & & & \\
\hline $\mathrm{C} 8$ & 57 & 70 & 2 & 30 & 33 & 31,67 & 27,33 & $-44,44 \%$ & $-60,95 \%$ \\
\hline & & & 3 & 33 & 29 & & & & \\
\hline
\end{tabular}

*Estes olhos não passaram por procedimento cirúrgico.

De acordo com Hardman \& Stanley (2001), a técnica de ciclofotocoagulação a laser, com laser diodo, é eficaz e os mesmos obtiveram sucesso em $92 \%$ dos casos. Já Sapienza \& Van Der Woerdt (2005) realizaram um estudo associando a ciclofotocoagulação a laser com gonioimplante (Ahmed) e obtiveram sucesso em $76 \%$ dos casos. Ambos corroboram com esse experimento, o qual obteve sucesso em 55\% dos casos, nos quais os cães ficaram com a PIO após a cirurgia abaixo de $30 \mathrm{mmHg}$ e acima de 15 $\mathrm{mmHg}$, em curto prazo. Somente dois olhos não tiveram a PIO diminuída abaixo de $30 \mathrm{mmHg}$, o que corresponde a $27,27 \%$ dos casos. Se fossemos considerar apenas a diminuição da PIO a eficácia da ciclofotocoagulação a laser seria de $100 \%$ nos casos desse experimento, já que em todos os olhos houve diminuição significativa da PIO após a cirurgia. A diferença destes dois trabalhos em comparação a este experimento foi a potência da energia utilizada. Hardman \& Stanley (2001) utilizaram o laser diodo com a potência de $1000 \mathrm{~mW}$ (milliwatts), enquanto Sapienza \& Van Der Woerdt (2005) realizaram o estudo em dois grupos utilizando energias diferentes, em um dos grupos a energia era considerada alta, entre $1500-1700 \mathrm{~mW}$, no outro grupo a energia era considerada baixa, ficando entre $800-1500 \mathrm{~mW}$. Independente da energia observou-se que o laser diodo é eficaz na redução da PIO, como já discutido anteriormente. A observação feita por Sapienza \& Van Der Woerdt (2005) é que, quando o laser diodo é utilizado com uma energia mais baixa há menos complicações pós-operatórias, porém a durabilidade 
do procedimento é menor, sendo de até seis meses. Enquanto em energias mais altas a cirurgia pode causar mais reações pós-operatórias, entretanto, a eficácia da cirurgia é observada por até 18 meses.

Outra discrepância observada entre os estudos é a diferença na quantidade de disparos dados. Neste experimento foram dados 12 tiros em cada olho operado. Enquanto no estudo de Hardman \& Stanley (2001) foram feitos 25 tiros e no estudo de Sapienza \& Van Der Woerdt (2005), o grupo de cães que foram operados com energia mais alta recebeu de 30 a 50 disparos, enquanto o grupo operado com energia mais baixa recebeu de 12 a 25 disparos. Nos dois trabalhos não houve discussão sobre a influência da quantidade de disparos sobre a eficácia do procedimento cirúrgico e/ou complicações pósoperatórias. Portanto, essa é uma questão que ainda merece ser estudada e discutida, já que há uma grande disparidade na quantidade de disparos que devem ser feitos durante o procedimento cirúrgico.

Esse estudo acompanhou os animais durante um mês, sendo considerado um período de tempo curto para avaliar a eficácia da ciclofotocoagulação a laser em longo prazo. Porém, de acordo com Hardman \& Stanley (2001), em 85\% dos casos a cirurgia foi eficaz, mantendo a PIO nos valores de normalidade, por mais de 6 meses, o que demonstra eficácia da mesma também em longo prazo. Em contrapartida, os procedimentos realizados com laser Nd:YAG obtiveram sucesso em 83\% dos casos durante 3 a 6 meses.

Segundo Hardman \& Stanley (2001) tanto o laser Nd:YAG quanto o laser diodo produzem efetiva ciclodestruição em tecidos, porém no caso do laser diodo a ciclodestruição ocorre com menor necessidade de energia, levando a uma menor reação inflamatória dos tecidos e menores complicações pós-operatórias. As seguintes complicações pós-operatórias foram observadas, tanto por Hardman \& Stanley (2001) quanto por Sapienza \& Van Der Woerdt (2005): catarata, ceratoconjuntivite seca, hifema, úlcera de córnea, phthisisbulbi. No OE do cão C3 observou-se um aumento da PIO no segundo retorno, com abrupta redução da mesma no terceiro retorno. Pode-se especular que ocorreu exacerbada formação de células inflamatórias, que obstruíram a saída do humor aquoso causando o aumento da PIO, e por seguinte houve a desobstrução da saída do aquoso levando à queda da PIO. Ademais, durante o acompanhamento dos cães para este estudo não foram observadas quaisquer complicações póscirúrgicas, mas, como já mencionado antes, os cães foram acompanhados por um curto período de tempo (30 dias), não podendo ser observadas complicações que aparecem ao longo do tempo.

\section{Conclusão}

O glaucoma é uma das oftalmopatias mais importantes da atualidade, sendo uma das doenças mais estudadas no mundo. Com os avanços da tecnologia, como o surgimento do laser oftalmológico, novas técnicas para o tratamento cirúrgico do glaucoma foram criadas, sendo cada vez mais eficazes, menos invasivas e com menos complicações pós-operatórias. Um exemplo de uma técnica relativamente nova é a realizada neste experimento, a ciclofotocoagulação a laser, que tem demonstrado ser uma das técnicas mais eficazes para o controle da PIO.

Dentro das condições em que foi realizado o presente experimento, conclui-se que a utilização do laser diodo infravermelho em pulso único na potência de $9000 \mathrm{~mW}$ é eficaz para o controle da PIO em cães. Todavia, por ser um estudo recente e com técnica cirúrgica pouco difundida na literatura, não havendo um padrão de quantidades de tiros disparados e níveis de energia ideal. Novos estudos devem ser realizados. Assim como estudos que avaliem se esta técnica pode ser considerada um tratamento definitivo para o glaucoma.

\section{Referências bibliográficas}

Hardman, C. \& Stanley, R. G. (2001). Diode laser transscleral cyclophotocoagulation for the treatment of primary glaucoma in 18 dogs: a retrospective study. Veterinary Ophthalmology, 4(3):209-215.

Laus, J. L. (2009). Oftalmologia clínica e cirúrgica em cães e em gatos. São Paulo: Roca.

Sapienza, J. S. \& Van Der Woerdt, A. (2005). Combined transscleral diode laser cyclophotocoagulation and Ahmed gonioimplantation in dogs with primary glaucoma: 51 cases (1996-2004). Veterinary Ophthalmology, 8(2):121-127.

Recebido: 10 de outubro, 2019 Aprovado: 19 de novembro, 2019.

Publicado: 10 de março, 2020.
Licenciamento: Este artigo é publicado na modalidade Acesso Aberto sob a licença Creative Commons Atribuição 4.0 (CC-BY 4.0), a qual permite uso irrestrito, distribuição, reprodução em qualquer meio, desde que o autor e a fonte sejam devidamente creditados. 